Macromolecular Nanotechnology

\title{
Antioxidant activity and photostability of $\alpha$-tocopherol/ $\beta$-cyclodextrin inclusion complex encapsulated electrospun polycaprolactone nanofibers
}

\author{
Zeynep Aytac, Tamer Uyar* \\ Institute of Materials Science E Nanotechnology, Bilkent University, Ankara 06800, Turkey \\ UNAM-National Nanotechnology Research Center, Bilkent University, Ankara 06800, Turkey
}

\section{A R T I C L E I N F O}

\section{Article history:}

Received 8 February 2016

Received in revised form 20 April 2016

Accepted 25 April 2016

Available online 26 April 2016

\section{Keywords}

$\alpha$-Tocopherol

$\beta$-Cyclodextrin

Polycaprolactone

Nanofiber

Antioxidant activity

Photostability

\begin{abstract}
A B S T R A C T
Cyclodextrin inclusion complexes (CD-ICs) can be encapsulated into electrospun nanofibers in order to achieve delivery systems having high surface area and highly porous nanofibrous structures. In this study, a well-known antioxidant molecule, $\alpha$-tocopherol $(\alpha-\mathrm{TC})$ (vitamin E) was chosen as an active agent for inclusion complexation with $\beta$ cyclodextrin. Polycaprolactone (PCL) nanofibers encapsulating $\alpha$-tocopherol $/ \beta$-cyclodex trin inclusion complex $(\alpha-\mathrm{TC} / \beta-\mathrm{CD}-\mathrm{IC})$ which has high antioxidant activity and photostability was produced via electrospinning (PCL/ $\alpha-\mathrm{TC} / \beta-\mathrm{CD}-\mathrm{IC}-\mathrm{NF}$ ). The formation of $\alpha-\mathrm{TC} / \beta-\mathrm{CD}-$ IC was confirmed by XRD. Phase solubility studies showed $A_{n}$-type complex formation between $\alpha$-TC and $\beta$-CD. SEM revealed that bead-free nanofibers were successfully produced from PCL $/ \alpha$-TC/ $\beta$-CD-IC system. PCL nanofibers encapsulating $\alpha$-TC without CD-IC was also produced for comparison (PCL/ $\alpha-\mathrm{TC}-\mathrm{NF})$. Antioxidant test results showed that $\mathrm{PCL} / \alpha-\mathrm{TC} / \beta-\mathrm{CD}-\mathrm{IC}-\mathrm{NF}$ had higher antioxidant activity as compared to $\mathrm{PCL} / \alpha-\mathrm{TC}-\mathrm{NF}$ in methanol:water (1:1) system due to the stabilization and solubility increment of $\alpha$-TC in the cavity of $\beta$-CD. PCL $/ \alpha-\mathrm{TC} / \beta-\mathrm{CD}$-IC-NF was more stable against UV-light when compared to PCL/ $\alpha$-TC-NF due to the presence of inclusion complexation. In brief, $\mathrm{PCL} / \alpha-\mathrm{TC} / \beta-\mathrm{CD}-\mathrm{IC}-$ $\mathrm{NF}$ with the advantages of having nanofibrous structure and encapsulating CD-ICs, may serve as a novel route for administration of $\alpha$-TC due to its higher antioxidant activity and better UV-light stability.
\end{abstract}

(c) 2016 Elsevier Ltd. All rights reserved.

\section{Introduction}

Cyclodextrins (CDs), nontoxic cyclic oligosaccharides (Fig. 1a), are produced by enzymatic degradation of starch. They consist of $\alpha$-1,4-linked glucopyranose units forming the truncated-cone shape molecular structure (Fig. 1b) [1]. The most widely used CD types are $\alpha-C D, \beta-C D$ and $\gamma$-CD having 6,7 and 8 glucopyranose units, respectively. The cavity size depends on the type (i.e. $\sim 6,8$ and $10 \AA$ for $\alpha-\mathrm{CD}, \beta-\mathrm{CD}$ and $\gamma-\mathrm{CD}$ ) while the depth of the above-mentioned $\mathrm{CDs}$ are identical and $\sim 8 \AA$ $[1,2]$. CDs are known for their ability to build up non-covalent inclusion complexes (ICs) with a variety of molecules in appropriate polarity and dimension by the substitution of high-enthalpy water molecules and guest molecule $[1,2]$. The formation of IC leads to considerable improvements in the properties of the guest molecules such as protection from evaporation,

\footnotetext{
* Corresponding author at: Institute of Materials Science \& Nanotechnology, Bilkent University, Ankara 06800, Turkey.

E-mail address: tamer@unam.bilkent.edu.tr (T. Uyar).
} 


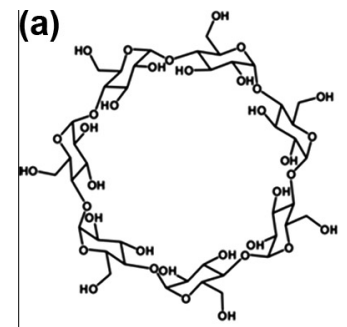

(d)

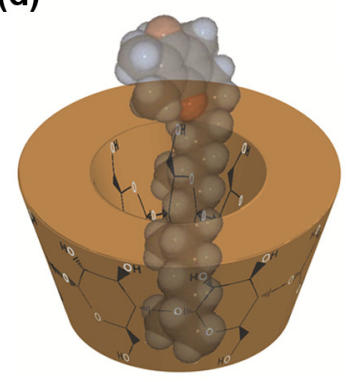

(b)

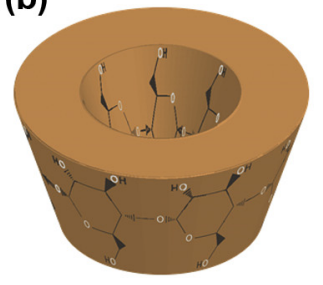

(e) (c)<smiles>[CH][C@H](CCCC[C@]1(C)CCc2c(C)c(O)c(C)c(C)c2O1)CCCC(C)C</smiles>

High voltage power supply

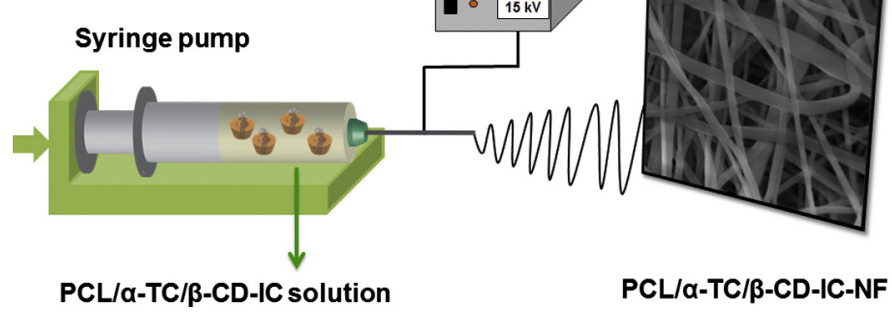

Fig. 1. The chemical structure of (a) $\beta$-CD, (b) schematic representation of $\beta-C D$, (c) the chemical structure of $\alpha$-TC, (d) formation of $\alpha$-TC/ $\beta$-CD-IC, and (e) electrospinning of nanofibers from PCL/ $\alpha-\mathrm{TC} / \beta-\mathrm{CD}-\mathrm{IC}$ solution.

degradation and oxidation; enhancing solubility, chemical stability; and controlling the release rate [1,3,4]. The most common application of CDs in the pharmaceutical area is related with the solubility, dissolution rate and bioavailability of drugs. Moreover, CDs are used not only for releasing of the drug molecules [5,6] but also masking the undesirable physicochemical properties in pharmaceutical applications [7-12].

$\alpha$-Tocopherol ( $\alpha$-TC) (Fig. 1c) which is the main component of vitamin $\mathrm{E}$ is generally used as a drug in wound dressing applications. But it is a poorly soluble compound and has other drawbacks like decomposing when exposed to oxygen, light, alkali $\mathrm{pH}$, trace of transition metal ions [13]. Recently, there has been a rising interest of using CDs in order to overcome limitations of antioxidants, antibacterials, drugs and provide advantages as mentioned above. The studies associated with IC of $\alpha$-TC with CDs were reported in the literature as well to increase solubility [14,15] and improve oxidative stability of foods [16]. In addition to these studies, there are studies in which $\alpha$-TC/CD-IC incorporated into polymeric films [17-19]. In the study of Siró et al., it was stated that diffusion rate of $\alpha$-TC from polymeric films reduced when it is in the cavity of CD [17]. In a study conducted by Koontz et al., the protection of the polymer from oxidative degradation and delay in the onset of oxidation of the packaged food during storage was achieved by inclusion complexation of $\alpha$-TC [18]. In another study of Koontz et al., it was deduced that polymeric films including CD-IC of $\alpha$-TC can be used for the extension of the shelf life of food products [19].

Electrospinning has received considerable attention during recent years because it is a convenient and efficient method to fabricate nanofibers possessing high surface to volume ratio and porous structure from variety of polymeric materials [2022]. In addition, functional electrospun nanofibers incorporated with active molecules [23-26] and CD-IC of different compounds incorporated electrospun nanofibers having the properties of both CD-IC and electrospun nanofibers were produced [27-40].

In this study, a commonly used antioxidant molecule, $\alpha$-TC was used for evaluating the capability of CD-IC incorporated nanofibers as a drug carrier system. Since the above-mentioned drawbacks of $\alpha$-TC including its hydrophobicity and instability against oxygen, light, alkali $\mathrm{pH}$, trace of transition metal ions limit its usage; here, we aimed to overcome these limitations that are common in most of the drugs by inclusion complexation with CDs [41]. Polycaprolactone (PCL) has slow degradation rate, biodegradability and non-toxicity; that's why it is widely used as carrier for drugs in wound dressing, drug delivery and pharmaceutical applications in the literature [42-45]. Therefore, it was selected as a polymeric matrix for this study.

\section{Materials and methods}

\subsection{Materials}

PCL (Mn 70,000-90,000 g/mol, Sigma Aldrich, Germany), $\alpha$-TC ( $\geqslant 96 \%$, Sigma Aldrich, Germany), $\beta$-CD, (gift from Wacker Chemie AG, Germany), formic acid (FA, extra pure 98-100\%, Sigma Aldrich, Germany), acetic acid (AA, extra pure 100\%, 
Sigma Aldrich, Germany), methanol (extra pure, Sigma Aldrich, Germany), methanol chromasol (Sigma Aldrich, Germany), potassium phosphate monobasic (Riedel de Haen, Germany), disodium hydrogen phosphate dodecahydrate (Riedel de Haen, Germany), sodium chloride (99.0-100.5\%, Sigma Aldrich, Germany), polysorbate 20 (Sigma Aldrich, Germany), 2,2-diphenyl1-picrylhydrazyl (DPPH, Sigma Aldrich, Germany) were purchased and used as received without any further purification. The water used in the experiments was distilled-deionized from a Millipore Milli-Q ultrapure water system.

\subsection{Preparation of solutions}

IC of $\alpha$-TC and $\beta$-CD ( $\alpha$-TC/ $\beta$-CD-IC) (Fig. 1d) was incorporated in PCL nanofibers (PCL/ $\alpha-\mathrm{TC} / \beta$-CD-IC-NF) by electrospinning (Fig. 1e). A free $\alpha$-TC without CD-IC was also incorporated in PCL nanofibers (PCL/ $\alpha$-TC-NF) for a comparative study. PCL/ $\alpha$-TC solution was prepared by dispersing $10 \% \alpha$-TC (w/w, with respect to polymer) in FA:AA $(1: 3, v / v)$ at room temperature (RT) and then $15 \% \mathrm{PCL}(\mathrm{w} / \mathrm{v})$ was added into the solution. Finally, the solution was stirred for $6 \mathrm{~h}$ before electrospinning. For producing PCL $/ \alpha-\mathrm{TC} / \beta-\mathrm{CD}-\mathrm{IC}-\mathrm{NF}, \alpha$-TC was used at $2: 1 \mathrm{M}$ ratio to $\beta$-CD ( $\alpha$-TC: $\beta-\mathrm{CD}$ ). 2:1 stoichiometry was preferred instead of using 1:1 in order to incorporate much more amount of $\alpha$-TC in the nanofiber matrix. PCL/ $\alpha-\mathrm{TC} / \beta$ CD-IC solution was prepared by dispersing $10 \% \alpha$-TC (w/w, with respect to polymer) in FA:AA $(1: 3, v / v)$ at RT, thereafter $13 \% \beta-\mathrm{CD}(\mathrm{w} / \mathrm{w}$, with respect to polymer) was added into the dispersion $\alpha$-TC. After stirring the solution at RT overnight, $15 \%$ PCL (w/v) was added and the solution was stirred for an additional $6 \mathrm{~h}$ before electrospinning. Vials were covered with aluminum foil during the stirring period to protect $\alpha$-TC from light-induced degradation. For comparison, we have also prepared the solution of PCL $(15 \%, w / v)$ in FA:AA $(1: 3, v / v)$ without $\alpha$-TC or $\alpha$-TC/ $\beta$-CD-IC and PCL nanofibers (PCL-NF) were obtained. The compositions of the above-mentioned solutions used for the electrospinning of nanofibers are summarized in Table 1.

\subsection{Electrospinning}

PCL, PCL $/ \alpha$-TC and PCL $/ \alpha$-TC/ $\beta$-CD-IC solutions were subsequently loaded into a $3 \mathrm{~mL}$ plastic syringe with metallic needle having an inner diameter of $0.8 \mathrm{~mm}$. The solutions in the syringes were pumped at a constant rate $(0.5 \mathrm{~mL} / \mathrm{h})$ by syringe pump (WPI, SP 101IZ). A fixed electrical potential of $15 \mathrm{kV}$ was applied from a high voltage power supply (AU Series, Matsusada Precision Inc.). Nanofibers were deposited on a grounded cylindrical metal covered with aluminum foil which was placed at a distance of $8 \mathrm{~cm}$ from the needle tip. The electrospinning of the nanofibers was carried out in an enclosed Plexiglas box at $22-26^{\circ} \mathrm{C}$ under $17-20 \%$ relative humidity.

\subsection{Phase solubility studies}

Phase solubility studies were carried out according to the previously described method [46]. Briefly, excess amount of $\alpha$ TC was added to $10 \mathrm{~mL}$ of aqueous solutions of $\beta-C D$ ranging in concentration from 0 to $0.016 \mathrm{M}$. Then, the suspensions were stirred in dark at RT for $12 \mathrm{~h}$ to achieve the equilibrium. After equilibrium was achieved, the samples were filtered through a membrane filter and analyzed spectrophotometrically at $292 \mathrm{~nm}$ (Varian, Cary 100). The experiment was carried out in triplicate and the phase diagram was drawn by plotting the molar concentration of $\alpha$-TC against the molar concentration of $\beta$ $\mathrm{CD}$. Each data point was the average of three determinations. The apparent stability constant $\left(\mathrm{K}_{\mathrm{s}}\right)$ was calculated from the phase solubility diagram with the assumption of 1:1 stoichiometry according to the following equation:

$$
K_{s}=\text { slope } / S_{0}(1-\text { slope })
$$

where $S_{0}$ is the intrinsic solubility of $\alpha$-TC.

\subsection{Crystalline structure of $\alpha-T C / \beta-C D-I C$}

X-ray diffraction (XRD) (PANalytical X'Pert powder diffractometer) patterns for as received $\beta$-CD, physical mixture of $\alpha$-TC and $\beta-\mathrm{CD}(\alpha-\mathrm{TC} / \beta-\mathrm{CD}-\mathrm{PM})$ and $\alpha-\mathrm{TC} / \beta-\mathrm{CD}-\mathrm{IC}$ were recorded in powder form to investigate the crystalline structure by applying $\mathrm{Cu} \mathrm{K} \alpha$ radiation at a range of $2 \theta=5-30^{\circ}$.

Table 1

The properties of the solutions used for electrospinning and morphological characteristics of the resulting nanofibers.

\begin{tabular}{llllllll}
\hline Solutions & $\begin{array}{l}\% \mathrm{PCL}^{\mathrm{a}} \\
(\mathrm{w} / \mathrm{v})\end{array}$ & $\begin{array}{l}\% \beta-\mathrm{CD}^{\mathrm{b}} \\
(\mathrm{w} / \mathrm{w})\end{array}$ & $\begin{array}{l}\% \alpha-\mathrm{TC}^{\mathrm{b}} \\
(\mathrm{w} / \mathrm{w})\end{array}$ & $\begin{array}{l}\text { Viscosity } \\
(\mathrm{cP})\end{array}$ & $\begin{array}{l}\text { Electrical conductivity } \\
(\mu \mathrm{S} / \mathrm{cm})\end{array}$ & $\begin{array}{l}\text { Average fiber } \\
\text { diameter }(\mathrm{nm})\end{array}$ & \begin{tabular}{l} 
Fiber morphology \\
\hline PCL
\end{tabular} \\
\hline 15 & - & - & 1795 & 4.7 & $255 \pm 130$ & Bead free nanofibers \\
PCL $\alpha-\mathrm{TC}$ & 15 & - & 10 & 1156 & 4.0 & $205 \pm 115$ & Bead free nanofibers \\
PCL $\alpha-\mathrm{TC} / \beta-\mathrm{CD}-\mathrm{IC}$ & 15 & 13 & 10 & 1631 & 4.3 & $345 \pm 140$ & Bead free nanofibers \\
\hline
\end{tabular}

\footnotetext{
${ }^{a}$ With respect to solvent (FA:AA, 1:3).
}

b With respect to polymer (PCL). 


\subsection{Viscosity and electrical conductivity measurement}

The viscosity measurements of PCL, PCL/ $\alpha$-TC and PCL/ $\alpha$-TC/ $\beta$-CD-IC solutions were performed at RT by Brookfield Viscometer DV-II + Pro equipped with cone/plate accessory using the spindle type CPE-51 and the electrical conductivity of the solutions were measured by Inolab ${ }^{\circledR}$ Multi 720-WTW at RT.

\subsection{Morphology analysis of nanofibers}

The morphological characterization of the electrospun PCL-NF, PCL/ $\alpha-\mathrm{TC}-\mathrm{NF}$ and PCL/ $\alpha-\mathrm{TC} / \beta-\mathrm{CD}-\mathrm{IC}-\mathrm{NF}$ was carried out by using scanning electron microscopy (SEM, FEI - Quanta 200 FEG). The nanofiber samples were prepared by mounting nanofibers on metal stubs using a double-sided copper adhesive tape and coated with $5 \mathrm{~nm}$ Au/Pd layer (PECS-682) prior to SEM observation to minimize charging of the samples. In order to determine diameter distributions and average fiber diameter (AFD) of the nanofibers, about 100 fibers were analyzed from SEM images.

\subsection{Thermal analysis of nanofibers}

The thermal properties of $\alpha$-TC (liquid), $\beta$-CD (powder), PCL-NF, PCL/ $\alpha$-TC-NF and PCL/ $\alpha$-TC/ $\beta$-CD-IC-NF were analyzed via thermogravimetric analysis (TGA, TA Q500, USA). For measurement, samples were heated from $25^{\circ} \mathrm{C}$ to $500{ }^{\circ} \mathrm{C}$ at a rate of $20^{\circ} \mathrm{C} / \mathrm{min}$ under $\mathrm{N}_{2}$ purge.

\subsection{Antioxidant activity}

The antioxidant activity of $\alpha$-TC after incorporation into PCL/ $\alpha$-TC-NF and PCL $/ \alpha$-TC/ $\beta$-CD-IC-NF were investigated via DPPH radical scavenging assay. PCL-NF was also used as control. PCL-NF (4.5 mg), PCL/ $\alpha-\mathrm{TC}-\mathrm{NF}(4.5 \mathrm{mg})$; and PCL/ $\alpha-\mathrm{TC} / \beta-$ CD-IC-NF ( $5 \mathrm{mg}$ ) having equivalent amount of $\alpha$-TC with PCL/ $\alpha$-TC-NF was immersed in $3 \mathrm{~mL}$ of $10^{-4} \mathrm{M}$ DPPH solution prepared in methanol and methanol:water (1:1). The mixture was incubated in dark at RT. The system reached equilibrium at the end of $30 \mathrm{~min}$ for methanol and $60 \mathrm{~min}$ for methanol:water (1:1) medium. Then, the absorbance of the mixtures was measured via UV-Vis NIR spectroscopy (Varian, Cary 5000) at the wavelength of 517 and 524 nm, respectively. The antioxidant activities (\%) were calculated according to the following equation:

$$
\text { Antioxidant activity }(\%)=\left(A_{\text {control }}-A_{\text {sample }}\right) / A_{\text {control }} * 100
$$

where $A_{\text {control }}$ and $A_{\text {sample }}$ represent the absorbance values of control DPPH solution and DPPH solution with PCL-NF, PCL/ $\alpha$ TC-NF or PCL $/ \alpha-T C / \beta-C D-I C-N F$, respectively. The experiments were carried out in triplicate and the results were presented as average \pm standard deviation values.

The cumulative amount of $\alpha$-TC released from PCL/ $\alpha$-TC-NF and PCL/ $\alpha-\mathrm{TC} / \beta-\mathrm{CD}-\mathrm{IC}-\mathrm{NF}$ were investigated for $24 \mathrm{~h}$ using total immersion method. The nanofibrous webs were individually immersed in $30 \mathrm{~mL}$ of phosphate buffer saline including $10 \%$ methanol (v/v) and $0.5 \%$ polysorbate $20(\mathrm{v} / \mathrm{v})\left(\right.$ PBMT) at $37^{\circ} \mathrm{C}$, and the solutions were stirred at $50 \mathrm{rpm}$. Methanol and polysorbate 20 were added to help the solubilisation of $\alpha$-TC in the releasing medium. At specified time intervals between 0 and $24 \mathrm{~h}, 0.5 \mathrm{~mL}$ of the sample solution was withdrawn and the solution was replenished with equal volume of fresh medium. The amount of $\alpha$-TC released from nanofibers into PBMT were determined via high-performance liquid chromatography (HPLC, Agilient 1200 Series) equipped with VWD UV detector set at $292 \mathrm{~nm}$. Chromatographic separation of $\alpha$-TC was achieved by C18 column (Agilient, column dimension: $4.6 \mathrm{~mm} \times 150 \mathrm{~mm}$, particle size: $5 \mu \mathrm{m}$ ) operating at $1 \mathrm{~mL} / \mathrm{min}$. The mobile phase for separation was 98:2 methanol:water $(\mathrm{v} / \mathrm{v})$ and the injection volume was $10 \mu \mathrm{L}$. The cumulative amount of $\alpha$-TC released from the samples at each immersion period was converted to concentration (ppm) according to the calibration curve obtained via the calibration samples prepared in PBMT. The release experiments were performed in triplicate and the results were reported as the average \pm standard deviation.

The antioxidant activity (\%) of $\alpha$-TC released from PCL/ $\alpha$-TC-NF and PCL/ $\alpha-\mathrm{TC} / \beta-\mathrm{CD}-\mathrm{IC}-\mathrm{NF}$ at the end of $24 \mathrm{~h}$ into PBMT at $37{ }^{\circ} \mathrm{C}$ was also investigated according to DPPH method. $0.5 \mathrm{~mL}$ of the sample withdrawn from the solutions of PCL/ $\alpha$-TC-NF and PCL $/ \alpha$-TC/ $\beta-C D-I C-N F$ were added to $2.5 \mathrm{~mL}$ of $10^{-4} \mathrm{M}$ DPPH prepared in methanol. After 30 min of incubation when the system reached equilibrium, the absorbance of the solutions was measured via UV-Vis NIR spectroscopy (Varian, Cary 5000) at the wavelength of $517 \mathrm{~nm}$. As mentioned above, antioxidant activities (\%) were calculated according to Eq. (2). These experiments were carried out in triplicate and the results were reported as the average \pm standard deviation.

\subsection{Photostability}

PCL/ $\alpha$-TC-NF and PCL/ $\alpha$-TC/ $\beta$-CD-IC-NF were tested for their photo-induced degradation. Nanofibers were cut into square shaped samples and exposed to UV light (300 W, Osram Ultra-Vitalux, E27/ES) from a distance of $30 \mathrm{~cm}$ for $65 \mathrm{~min}$. The photostability of $\alpha$-TC in PCL/ $\alpha$-TC-NF and PCL/ $\alpha$-TC/ $\beta$-CD-IC-NF was investigated by performing in vitro release test according to procedures explained in the release study part. The results were compared with the results obtained from release studies 
of non-treated nanofibers. These experiments were conducted in triplicate and the results were given as average \pm standard values.

\section{Results and discussion}

\subsection{Phase solubility studies}

The phase solubility diagram of $\alpha$-TC and $\beta$-CD is presented in Fig. 2. The solubility curve can be classified as $A_{n}$ type [46]. The solubility of $\alpha$-TC increased linearly as the concentration of $\beta$-CD in the range from 0 to $0.012 \mathrm{M}$. When the $\beta$-CD concentration exceeds $0.012 \mathrm{M}$, less soluble complex formation was observed and the solubility of $\alpha$-TC started to decrease starting from that point. So, the solubility of complexes was higher than free $\alpha-\mathrm{TC}$. $\mathrm{K}_{\mathrm{s}}$ was calculated as $102 \mathrm{M}^{-1}$ from initial linear portions of the phase solubility diagram according to Eq. (1) and this value was an indication of weak interaction between $\alpha$-TC and $\beta$-CD [47].

\subsection{Crystalline structure of $\alpha-T C / \beta-C D-I C$}

XRD patterns of $\beta-\mathrm{CD}, \alpha-\mathrm{TC} / \beta-\mathrm{CD}-\mathrm{PM}$ and $\alpha$-TC/ $\beta$-CD-IC are given in Fig. 3. Since $\alpha$-TC is a liquid compound at RT, XRD analysis was not run for pure $\alpha$-TC. The cage type crystal packing in which cavity of each CD molecule is blocked by other $\mathrm{CD}$ molecules is seen in as received native CDs. Therefore, as received native CDs have characteristic peaks in the range of $2 \theta=5-30^{\circ}[48]$. When the guest molecules are included in the cavity of native CDs, crystalline channel type complexes are yielded. In the XRD pattern of $\alpha-\mathrm{TC} / \beta$-CD-PM, cage type crystal packing of $\beta$-CD was still observed suggesting the absence of IC between the guest molecule and CDs. Instead of cage type crystalline peaks of as received $\beta-C D$, the new crystalline peaks were observed in the XRD pattern of $\alpha$-TC/ $\beta$-CD-IC. This result showed the new crystalline phase formation in $\alpha$ $\mathrm{TC} / \beta-\mathrm{CD}-\mathrm{IC}$ and therefore it might be deduced that IC was successfully formed between $\alpha-\mathrm{TC}$ and $\beta-\mathrm{CD}$.

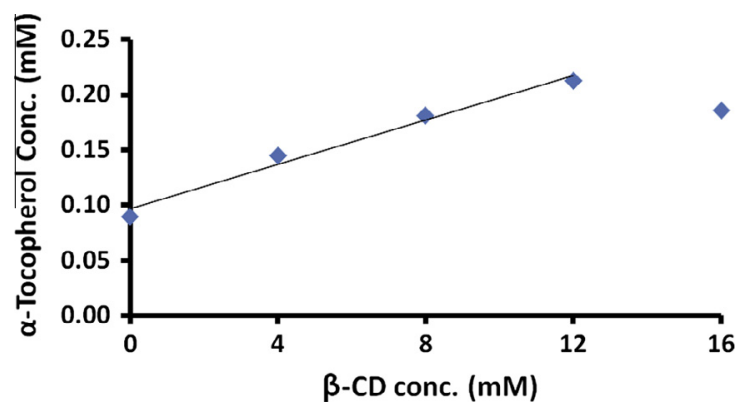

Fig. 2. Phase solubility diagram of $\alpha-\mathrm{TC} / \beta-\mathrm{CD}$ system in water.

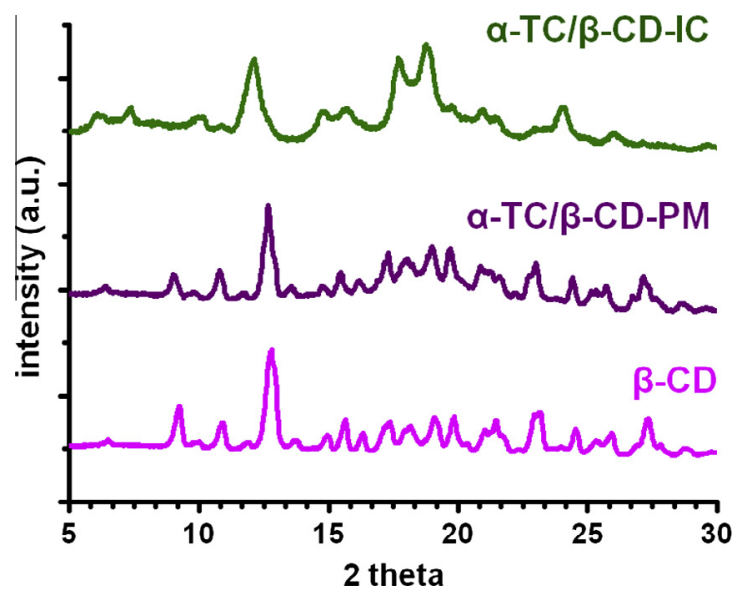

Fig. 3. XRD patterns of $\beta-C D, \alpha-T C / \beta-C D-P M$ and $\alpha-T C / \beta-C D-I C$. 

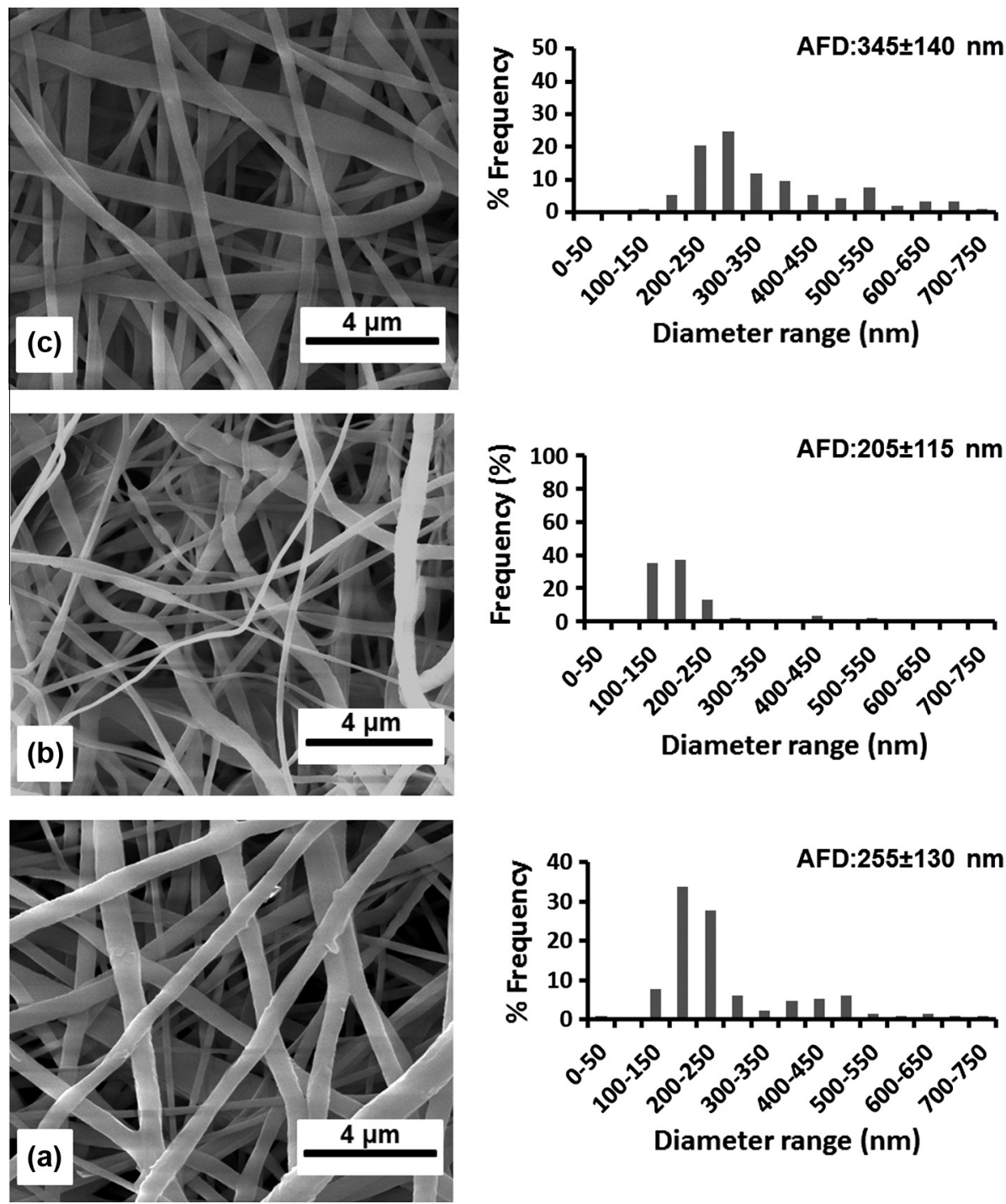

Fig. 4. SEM images and fiber diameter distributions with AFD of electrospun nanofibers obtained from solutions of (a) PCL, (b) PCL/ $\alpha$-TC, and (c) PCL/ $\alpha$-TC/ $\beta$-CD-IC. 100 fibers were analyzed from SEM images to calculate AFD.

\subsection{Morphology analysis of nanofibers}

SEM images and AFD along with fiber distribution of PCL-NF, PCL/ $\alpha$-TC-NF and PCL/ $\alpha-T C / \beta-C D-I C-N F$ are shown in Fig. 4ac. Bead-free PCL-NF with $255 \pm 130 \mathrm{~nm}$ average diameter was produced. Then, $\alpha$-TC or $\alpha$-TC/ $\beta$-CD-IC was incorporated into PCL solution and bead-free PCL/ $\alpha$-TC-NF and PCL/ $\alpha$-TC/ $\beta$-CD-IC-NF having $205 \pm 115 \mathrm{~nm}$ and $345 \pm 140 \mathrm{~nm}$ average diameter were obtained, respectively. Employing beaded nanofibers to incorporate various additives could be problematic, because beads might encapsulate some of the additives. In addition to that, inhomogeneous distribution of the additive throughout the nanofibers could be observed. In order to investigate how the viscosity and electrical conductivity affect the fiber diameter, viscosity and electrical conductivity measurements were performed and the results are shown with morphological findings and AFD of the resulting electrospun nanofibers in Table 1. The variations in AFD among the samples are most likely due to the differences in the viscosity and electrical conductivity of the solutions. The lower AFD of PCL/ $\alpha$-TC-NF compared to PCL-NF might be due to the lower viscosity of PCL/ $\alpha$-TC solution than PCL solution. Since lower viscosity induces the polymer jet to be stretch and elongate readily thus nanofibers with thinner diameter is obtained [21]. The viscosity of PCL/ $\alpha-\mathrm{TC} / \beta-\mathrm{CD}-$ IC and PCL solutions was similar, whereas the electrical conductivity of PCL/ $\alpha-T C / \beta-C D-I C$ solution was lower than PCL solution. The lower electrical conductivity is known to cause less stretching of the solution, and the diameter of the nanofiber increases [21]. Therefore, AFD of PCL/ $\alpha$-TC/ $\beta$-CD-IC-NF was higher than PCL-NF. Moreover, we did not observe any noticeable 


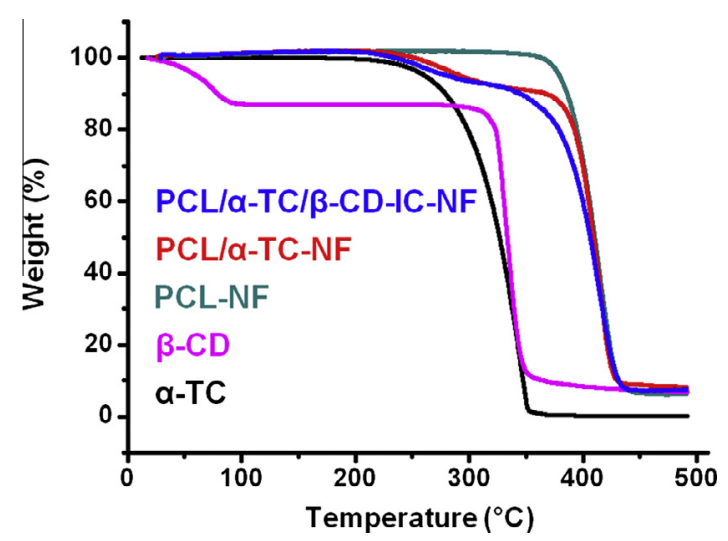

Fig. 5. TGA thermograms of $\alpha-\mathrm{TC}, \beta-\mathrm{CD}, \mathrm{PCL}-\mathrm{NF}, \mathrm{PCL} / \alpha-\mathrm{TC}-\mathrm{NF}$, and PCL/ $\alpha-\mathrm{TC} / \beta-\mathrm{CD}-\mathrm{IC}-\mathrm{NF}$.

difference in the morphology of nanofibers after the incorporation of both $\alpha$-TC and $\alpha$-TC/ $\beta$-CD-IC. To conclude, bead-free nanofibers were successfully obtained from PCL $/ \alpha-\mathrm{TC}$ and $\mathrm{PCL} / \alpha-\mathrm{TC} / \beta-\mathrm{CD}-\mathrm{IC}$ solutions.

\subsection{Thermal analysis of nanofibers}

TGA measurements for $\alpha$-TC, $\beta-C D$, PCL-NF, PCL/ $\alpha$-TC-NF and PCL/ $\alpha-T C / \beta-C D-I C-N F$ are given in Fig. 5 . The thermal degradation of $\alpha$-TC started at about $200^{\circ} \mathrm{C}$ and continued till $380^{\circ} \mathrm{C}$; whereas the thermal degradation of PCL is between $350{ }^{\circ} \mathrm{C}$ and $450{ }^{\circ} \mathrm{C}$. TGA thermogram of PCL/ $\alpha$-TC-NF showed two weight losses: the initial weight loss starting from $200^{\circ} \mathrm{C}$ was due to the degradation of $\alpha$-TC and the major weight loss between $350^{\circ} \mathrm{C}$ and $450^{\circ} \mathrm{C}$ corresponded to the degradation of PCL. The amount of $\alpha$-TC was determined as $\sim 9 \%$ from the TGA thermogram of PCL/ $\alpha$-TC-NF and this amount was in a good agreement with the initial amount of $\alpha$-TC that was used. The weight losses of $\beta$-CD were observed below $100{ }^{\circ} \mathrm{C}$ and above $275{ }^{\circ} \mathrm{C}$ corresponding to the water loss and the main thermal degradation of $\beta-\mathrm{CD}$, respectively. For PCL/ $\alpha-\mathrm{TC} / \beta-\mathrm{CD}-\mathrm{IC}-\mathrm{NF}$, two stages of weight loss was observed in TGA thermogram. The first weight loss that is starting at around $200^{\circ} \mathrm{C}$ belongs to $\alpha$-TC decomposition. The second weight loss starting from $300^{\circ} \mathrm{C}$ in $\mathrm{PCL} / \alpha-\mathrm{TC} / \beta-\mathrm{CD}-\mathrm{IC}-\mathrm{NF}$ is attributed to the decomposition of $\beta$-CD and PCL. Thus, the thermal stability of $\alpha$-TC did not change by the formation of inclusion complexation [16]. Finally, the amount of $\alpha$-TC was calculated as $\sim 8 \%$ from the TGA thermogram of PCL $/ \alpha$-TC/ $\beta$-CD-IC-NF and this was consistent with the initial amount of $\alpha$-TC used for PCL $/ \alpha-\mathrm{TC} / \beta$-CD-IC-NF. It can be concluded from TGA results that the thermal stability of $\alpha$-TC did not reduce by complexation.

\subsection{Antioxidant activity}

DPPH is a stable radical which can easily be scavenged by an antioxidant compound and therefore it is a widely used compound to test the free radical scavenging of antioxidant molecules. The mechanism of the assay is based on the reduction of DPPH through an antioxidant that is the donor of electron and hydrogen atom [49,50]. $\alpha$-TC which is an antioxidant molecule transforms DPPH radical into its reduced form, 2,2-diphenyl-1-picrylhydrazine (DPPH-H) by losing its phenolic hydrogen. Thus free radical character of DPPH is neutralized and the purple colour of the solution turns to yellow. DPPH is soluble in alcohols and using long-chain alcohols results in dissociation of the complex even at low concentrations. So, DPPH is usually dissolved in short-chain alcohols such as methanol and ethanol. Since hydrophobic (relatively low polarity) medium of alcohols reduce the hydrophobic driving force for complexation, they are not suitable for CD-ICs. That's why; the amount of methanol was reduced while dissolving the DPPH, because DPPH precipitates if the methanol amount is less than $50 \%$. So, we have decided to use methanol:water $(1: 1)$ medium in addition to methanol for evaluating antioxidant activity of guest molecules in the cavity of CDs. Although PCL-NF does not include any antioxidant molecule, it had $10 \pm 4 \%$ and $11 \pm 3 \%$ antioxidant activity in methanol and methanol:water (1:1) systems, respectively (Table 2). This activity might be related with the absorption of DPPH due to the high surface area of the nanofibers providing more contact with the medium. The antioxidant activity of PCL/ $\alpha-\mathrm{TC}-\mathrm{NF}$ and PCL/ $\alpha-\mathrm{TC} / \beta-\mathrm{CD}-\mathrm{IC}-\mathrm{NF}$ in methanol were calculated as $96 \pm 0 \%$ and $96 \pm 0 \%$; whereas PCL/ $\alpha$-TC-NF and PCL/ $\alpha$-TC/ $\beta$-CD-IC-NF have exhibited $22 \pm 1 \%$ and $57 \pm 2 \%$ antioxidant activity in methanol:water (1:1) medium (Table 2). As seen from the results, the antioxidant activity of PCL/ $\alpha$-TC/ $\beta$-CD-IC-NF and PCL/ $\alpha$-TC-NF was similar in methanol. Since $\alpha$-TC might release quickly from CD cavity and PCL nanofibers toward methanol most probably due to the high solubility of $\alpha$-TC in methanol. Therefore, the difference in the antioxidant activity of PCL/ $\alpha$-TC/ $\beta$-CD-IC-NF in two medium might be related with the position of $\alpha$-TC according to the cavity of $\beta$-CD. The antioxidant activity of $\alpha$-TC is almost 35\% higher in PCL/ $\alpha-\mathrm{TC} / \beta-\mathrm{CD}-\mathrm{IC}-\mathrm{NF}$ compared to PCL/ $\alpha$-TC-NF in methanol:water $(1: 1)$, the improvement is likely due to the solubility increment and stabilization of $\alpha$-TC in the CD cavity [51]. The colour of DPPH solution prepared in 
Table 2

The antioxidant activity (\%) of electrospun nanofibrous webs.

\begin{tabular}{|c|c|c|c|}
\hline Nanofibers & $\begin{array}{l}\text { Antioxidant activity } \\
\text { in methanol (\%) }\end{array}$ & $\begin{array}{l}\text { Antioxidant activity in } \\
\text { methanol:water }(1: 1)(\%)\end{array}$ & $\begin{array}{l}\text { Antioxidant activity } \\
\text { after release experiment }\end{array}$ \\
\hline PCL-NF & $10 \pm 4$ & $11 \pm 3$ & - \\
\hline $\mathrm{PCL} / \alpha-\mathrm{TC}-\mathrm{NF}$ & $96 \pm 0$ & $22 \pm 1$ & $26 \pm 3$ \\
\hline $\mathrm{PCL} / \alpha-\mathrm{TC} / \beta-\mathrm{CD}-\mathrm{IC}-\mathrm{NF}$ & $96 \pm 0$ & $57 \pm 2$ & $32 \pm 5$ \\
\hline
\end{tabular}

methanol turned from purple to yellow for both PCL/ $\alpha$-TC-NF and PCL $/ \alpha-\mathrm{TC} / \beta-\mathrm{CD}-\mathrm{IC}-\mathrm{NF}$. This result showed that there is no more DPPH molecule to be deactivated in the system. However, the colour of the solution in which PCL-NF was immersed did not change noticeably. As regards to DPPH solutions prepared in methanol:water (1:1), the colour of the solution in which PCL/ $\alpha$-TC-NF was immersed turned from purple to lighter purple, and the lightest purple colour was observed in the solution of PCL $/ \alpha-\mathrm{TC} / \beta-\mathrm{CD}-\mathrm{IC}-\mathrm{NF}$. As a result, PCL $/ \alpha-\mathrm{TC} / \beta-\mathrm{CD}-\mathrm{IC}-\mathrm{NF}$ exhibited better antioxidant activity than $\mathrm{PCL} / \alpha-$ TC-NF in methanol:water (1:1) system, but the antioxidant activity of PCL/ $\alpha$-TC-NF and PCL/ $\alpha$-TC/ $\beta$-CD-IC-NF was similar in methanol system.

Fig. 6 shows the release of $\alpha$-TC from PCL/ $\alpha$-TC-NF and PCL/ $\alpha-\mathrm{TC} / \beta-\mathrm{CD}-\mathrm{IC}-\mathrm{NF}$ at $37{ }^{\circ} \mathrm{C}$ into PBMT for $24 \mathrm{~h}$ and the release profile can be analyzed basically in two stages. Initial rapid release was up to $4 \mathrm{~h}$; then the release rate slowed down and followed by a steady release stage, thus no more $\alpha$-TC released further. The rate of $\alpha$-TC release from PCL $/ \alpha$-TC/ $\beta$-CD-ICNF was found to be similar with PCL/ $\alpha$-TC-NF in the initial period $(4 \mathrm{~h})$. The total amount of released $\alpha$-TC was $23 \pm 2 \mathrm{ppm}$ and $18 \pm 3 \mathrm{ppm}$ from PCL/ $\alpha$-TC-NF and PCL $/ \alpha-\mathrm{TC} / \beta$-CD-IC-NF, respectively. The difference in total release of $\alpha-$ TC could be due to the lower percentage of $\alpha$-TC in PCL $/ \alpha-\mathrm{TC} / \beta-\mathrm{CD}-\mathrm{IC}-\mathrm{NF}$ in comparison with PCL/ $\alpha$-TC-NF. Additionally, fibrous structure of nanofibers was still kept after the release experiments as seen from representative SEM images in inset of Fig. 6.

The antioxidant activity of PCL/ $\alpha-\mathrm{TC}-\mathrm{NF}$ and PCL/ $\alpha-\mathrm{TC} / \beta-\mathrm{CD}-\mathrm{IC}-\mathrm{NF}$ was calculated after release experiment as well. As seen from Table 2 , the antioxidant activity of PCL/ $\alpha$-TC-NF and PCL $/ \alpha-\mathrm{TC} / \beta-\mathrm{CD}-\mathrm{IC}-\mathrm{NF}$ was calculated to be $26 \pm 3 \%$ and $32 \pm 5 \%$, respectively. We could not obtain high antioxidant activity values like we obtained in previously mentioned systems due to the different procedure of antioxidant activity tests. Because taken samples was diluted firstly by $30 \mathrm{~mL}$ of PBMT and then with $2.5 \mathrm{~mL}$ of DPPH. Although PCL/ $\alpha-\mathrm{TC} / \beta-\mathrm{CD}$-IC-NF released less amount of $\alpha$-TC than PCL/ $\alpha$-TC-NF in the release experiment (Fig. 6), its antioxidant activity was higher compared to PCL/ $\alpha$-TC-NF. Increment in the solubility of PCL/ $\alpha$-TC/ $\beta$-CD-IC-NF as shown in phase solubility diagram and stabilization of $\alpha$-TC in CD cavity was most likely the reasons for higher antioxidant activity. In addition, the colour of the solution in which PCL/ $\alpha-\mathrm{TC} / \beta-\mathrm{CD}-\mathrm{IC}-\mathrm{NF}$ was immersed was yellowish; whereas the colour of $\mathrm{PCL} / \alpha-\mathrm{TC}-\mathrm{NF}$ was purplish. It is also important to mention that, antioxidant activity results showed that incorporated $\alpha$-TC retained its radical scavenging ability in PCL $/ \alpha$-TC-NF and PCL $/ \alpha$-TC/ $\beta$-CD-IC-NF despite the existence of the interactions between $\alpha$-TC and PCL nanofibers after electrospinning [52].

\subsection{Photostability}

The photodegradation might reduce the bioavailability of drugs by producing toxic photodegradation products. Therefore, providing high photostability of drugs is significant for pharmaceutical industry. On the other hand, the additive compounds which are used in sunscreen formulations as an antioxidant agent scavenge free radicals. However, it was previously

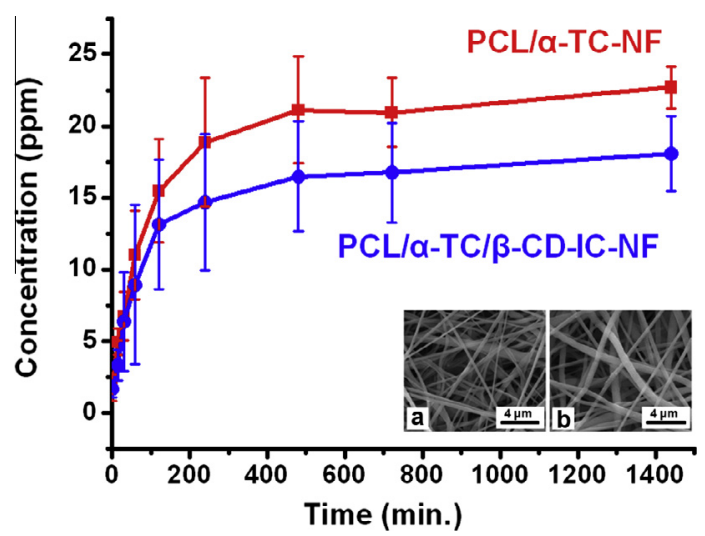

Fig. 6. The cumulative release of $\alpha$-TC from PCL $/ \alpha-T C-N F$ and PCL $/ \alpha-T C / \beta-C D-I C-N F$ at $37{ }^{\circ} \mathrm{C}$ into PBMT for $24 \mathrm{~h}(\mathrm{n}=3$ ) and SEM images of $(a)$ PCL $/ \alpha-T C-N F$ and (b) PCL/ $\alpha-\mathrm{TC} / \beta-\mathrm{CD}-\mathrm{IC}-\mathrm{NF}$ after release experiment. The error bars in the figure represent the standard deviation. 

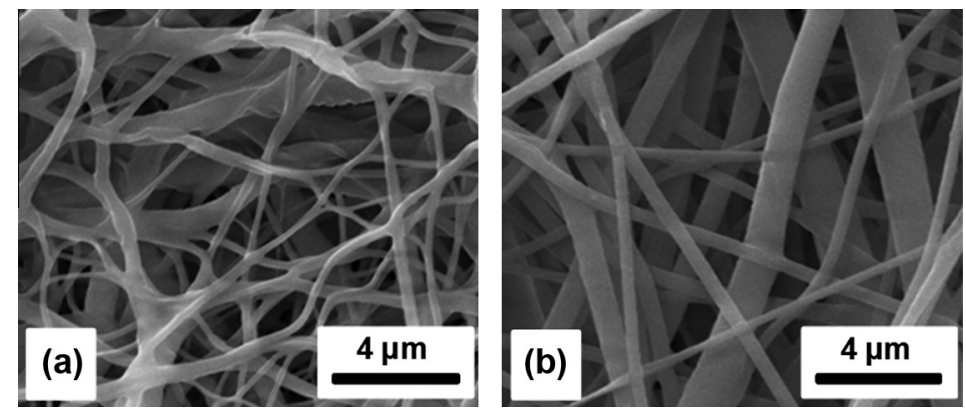

Fig. 7. SEM images of UV-treated (a) PCL/ $\alpha-\mathrm{TC}-\mathrm{NF}$ and (b) PCL $/ \alpha-\mathrm{TC} / \beta-\mathrm{CD}-\mathrm{IC}-\mathrm{NF}$.

reported that $\alpha$-TC degrades when it is exposed to UV light [13]. So, the photostability of $\alpha$-TC is of vital importance in its applications as a drug and an antioxidant. Of different methods to inhibit photodegradation products, side effects; and to maintain activity of antioxidant molecules, inclusion complexation with CDs is a promising approach. Since it is a known fact that CDs have protecting ability for compounds against oxidation, volatility, visible or UV light, heat [1]. According to the release of $\alpha$-TC from non-treated nanofibers, the percent (\%) of $\alpha$-TC that had been released from UV-treated nanofibers was $91 \pm 1 \%$ of non-treated nanofibers; whereas UV-treated PCL $/ \alpha$-TC/ $\beta$-CD-IC-NF released $97 \pm 0 \%$ of $\alpha$-TC in non-treated nanofiber. Therefore, the protection of $\alpha$-TC from UV light is more efficient (about 6\%) in PCL/ $\alpha$-TC/ $\beta$-CD-IC-NF most probably due to inclusion complexation between $\alpha$-TC and $\beta$-CD. Since CD-ICs provide a more apolar environment that reduces the photolytic reaction [53]. In addition, screening effect also gives rise to the protection of photostability owing to the prevention of passing of light toward the guest molecule [53]. As seen from the representative SEM images of UV-treated PCL/ $\alpha$-TC$\mathrm{NF}$ and $\mathrm{PCL} / \alpha-\mathrm{TC} / \beta-\mathrm{CD}-\mathrm{IC}-\mathrm{NF}$ in Fig. 7 , nanofibers maintained their fibrous structure, thus UV light did not deteriorate the fibrous morphology of PCL/ $\alpha-\mathrm{TC}-\mathrm{NF}$ and PCL/ $\alpha-\mathrm{TC} / \beta-\mathrm{CD}-\mathrm{IC}-\mathrm{NF}$ up to $65 \mathrm{~min}$ under the applied conditions.

\section{Conclusion}

Incorporating CD-ICs of drugs with electrospun nanofibers might be a good alternative for drug carrier systems. In this study, $\alpha$-TC was used as a model hydrophobic drug, PCL/ $\alpha$-TC-NF and PCL/ $\alpha$-TC/ $\beta$-CD-IC-NF was produced through electrospinning method. The successful formation of $\alpha-\mathrm{TC} / \beta-\mathrm{CD}-\mathrm{IC}$ was shown by using XRD. The solubility of $\alpha$-TC increased with the addition certain amount of $\beta-\mathrm{CD}$ as revealed from phase solubility diagram. PCL/ $\alpha-\mathrm{TC} / \beta-\mathrm{CD}-\mathrm{IC}-\mathrm{NF}$ was superior to PCL/ $\alpha$ TC-NF due to the presence of CD-IC providing higher oxidative stability and stronger photostability of $\alpha$-TC. As a result, PCL/ $\alpha-\mathrm{TC} / \beta-\mathrm{CD}-\mathrm{IC}-\mathrm{NF}$ could be an alternative material for the topical delivery of poorly soluble drugs.

\section{Acknowledgements}

Dr. Uyar acknowledges The Scientific and Technological Research Council of Turkey (TUBITAK)-Turkey (Project no. $111 \mathrm{M} 459$ ) for funding of the research. The Turkish Academy of Sciences - Outstanding Young Scientists Award Program (TUBA-GEBIP)-Turkey is also acknowledged for partial funding of the research. Z. Aytac thanks to TUBITAK-BIDEB and TUBITAK (project no. 111M459 and 213M185) for the PhD scholarship.

\section{References}

[1] E.M.M. Del Valle, Cyclodextrins and their uses: a review, Process Biochem. 39 (2004) 1033-1046.

[2] J. Szejtli, Introduction and general overview of cyclodextrin chemistry, Chem. Rev. 98 (1998) 1743-1753.

[3] A.R. Hedges, Industrial applications of cyclodextrins, Chem. Rev. 98 (1998) 2035-2044.

[4] J. Szejtli, L. Szente, Elimination of bitter, disgusting tastes of drugs and foods by cyclodextrins, Eur. J. Pharm. Biopharm. 61 (2005) 115-125.

[5] C. Rodriguez-Tenreiro, C. Alvarez-Lorenzo, A. Rodriguez-Perez, A. Concheiro, J.J. Torres-Labandeir, Estradiol sustained release from high affinity cyclodextrin hydrogels, Eur. J. Pharm. Biopharm. 66 (2007) 55-62.

[6] S. Swaminathan, L. Pastero, L. Serpe, F. Trotta, P. Vavia, D. Aquilano, M. Trotta, G.P. Zara, C. Roberta, Cyclodextrin-based nanosponges encapsulating camptothecin: physicochemical characterization, stability and cytotoxicity, Eur. J. Pharm. Biopharm. 74 (2010) 193-201.

[7] N. Funasaki, R. Kawaguchi, S. Hada, S. Neya, Ultraviolet spectroscopic estimation of microenvironments and bitter tastes of oxyphenonium bromide in cyclodextrin solutions, J. Pharm. Sci. 88 (1999) 759-762.

[8] F.M. Mady, A.E. Abou-Taleb, K.A. Khaled, K. Yamasaki, D. Iohara, K. Taguchi, M. Anraku, F. Hirayama, K. Uekama, M. Otagiri, Evaluation of carboxymethyl- $\beta$-cyclodextrin with acid function: improvement of chemical stability, oral bioavailability and bitter taste of famotidine, Int. J. Pharm. 397 (2010) $1-8$.

[9] J. Manosroi, M.G. Apriyani, K. Foe, A. Manosroi, Enhancement of the release of azelaic acid through the synthetic membranes by inclusion complex formation with hydroxypropyl- $\beta$-cyclodextrin, Int. J. Pharm. 293 (2005) 235-240.

[10] G. Piel, M. Piette, V. Barillaro, D. Castagne, B. Evrard, L. Delattre, Betamethasone-in-cyclodextrin-in-liposome: the effect of cyclodextrins on encapsulation efficiency and release kinetics, Int. J. Pharm. 312 (2006) 75-82.

[11] W. Samprasit, P. Akkaramongkolporn, T. Ngawhirunpat, T. Rojanarata, R. Kaomongkolgit, P. Opanasopit, Fast releasing oral electrospun PVP/CD nanofiber mats of taste-masked meloxicam, Int. J. Pharm. 487 (2015) 213-222. 
[12] K. Woertz, C. Tissen, P. Kleinebudde, J. Breitkreutz, Rational development of taste masked oral liquids guided by an electronic tongue, Int. J. Pharm. 400 (2010) 114-123.

[13] P.M. Bramley, I. Elmadfa, A. Kafatos, J.F. Kelly, Y. Manios, H.E. Roxborough, W. Schuch, P.J.A. Sheehy, K.H. Wagner, Vitamin E, J. Sci. Food Agric. 80 (2000) 913-938.

[14] S.E. Celik, M. Ozyurek, K. Guclu, R. Apak, CUPRAC total antioxidant capacity assay of lipophilic antioxidants in combination with hydrophilic antioxidants using the macrocyclic oligosaccharide methyl $\beta$-cyclodextrin as the solubility enhancer, React. Funct. Polym. 67 (2007) 1548-1560.

[15] Y. Sueishi, M. Hori, N. Inazumi, Characterization of inclusion complex of vitamin E compound with 2,6-di-O-methylated $\beta$-cyclodextrin as the solubility enhancer and its kinetic determination for radical scavenging ability. J. Incl. Phenom. Macrocycl. Chem. 72 (2012) 467-472.

[16] J.L. Koontz, J. Marcy, S.F. O'Keefe, S.E. Duncan, Cyclodextrin inclusion complex formation and solid-state characterization of the natural antioxidants $\alpha-$ tocopherol and quercetin, J. Agric. Food Chem. 57 (2009) 1162-1171.

[17] I. Siró, É. Fenyvesi, L. Szente, B. De Meulenaer, F. Devlieghere, J. Orgoványi, J. Sényi, J. Barta, Food Addit. Contam. 23 (2006) 845-853.

[18] J.L. Koontz, J.E. Marcy, S.F. O'Keefe, S.E. Duncan, T.E. Long, R.D. Moffitt, Polymer processing and characterization of LLDPE films loaded with $\alpha-$ tocopherol, quercetin, and their cyclodextrin inclusion complexes, J. Appl. Polym. Sci. 117 (2010) 2299-2309.

[19] J.L. Koontz, R.D. Moffitt, J.E. Marcy, S.F. O'Keefe, S.E. Duncan, T.E. Long, Controlled release of $\alpha$-tocopherol, quercetin, and their cyclodextrin inclusion complexes from linear low-density polyethylene (LLDPE) films into a coconut oil model food system, Food Addit. Contam. - Part A Chem., Anal., Control, Expo. Risk Assess. 27 (2010) 1598-1607.

[20] A. Greiner, J.H. Wendorff, Electrospinning: a fascinating method for the preparation of ultrathin fibers, Angew. Chem.-Int. Ed. 46 (2007) 5670-5703.

[21] S. Ramakrishna, An Introduction to Electrospinning and Nanofibers, World Scientific Pub Co Inc., Singapore, 2005.

[22] J.H. Wendorff, S. Agarwal, A. Greiner, Electrospinning: Materials, Processing, and Applications, Wiley-VCH, Weinheim, 2012.

[23] R.A. Thakur, C.A. Florek, J. Kohn, B.B. Michniak, Electrospun nanofibrous polymeric scaffold with targeted drug release profiles for potential application as wound dressing, Int. J. Pharm. 364 (2008) 87-93.

[24] X. Wang, D.G. Yu, X.Y. Li, S.W.A. Bligh, G.R. Williams, Electrospun medicated shellac nanofibers for colon-targeted drug delivery, Int. J. Pharm. 490 (2015) 384-390.

[25] X. Xu, X. Chen, P. Ma, X. Wang, X. Jing, The release behavior of doxorubicin hydrochloride from medicated fibers prepared by emulsion-electrospinning, Eur. J. Pharm. Biopharm. 70 (2008) 165-170.

[26] D.G. Yu, L.D. Gao, K. White, C. Branford-White, W.Y. Lu, L.M. Zhu, Multicomponent amorphous nanofibers electrospun from hot aqueous solutions of a poorly soluble drug, Pharm. Res. 27 (2010) 2466-2477.

[27] Z. Aytac, S.Y. Dogan, T. Tekinay, T. Uyar, Release and antibacterial activity of allyl isothiocyanate/ $\beta$-cyclodextrin complex encapsulated in electrospun nanofibers, Colloids Surf. B: Biointerfaces 120 (2014) 125-131.

[28] F. Kayaci, Y. Ertas, T. Uyar, Enhanced thermal stability of eugenol by cyclodextrin inclusion complex encapsulated in electrospun polymeric nanofibers, J. Agric. Food Chem. 61 (2013) 8156-8165.

[29] F. Kayaci, O.C.O. Umu, T. Tekinay, T. Uyar, Antibacterial electrospun poly (lactic acid) (PLA) nanofibrous webs incorporating triclosan/cyclodextrin inclusion complexes, J. Agric. Food Chem. 61 (2013) 3901-3908.

[30] F. Kayaci, H.S. Sen, E. Durgun, T. Uyar, Functional electrospun polymeric nanofibers incorporating geraniol-cyclodextrin inclusion complexes: high thermal stability and enhanced durability of geraniol, Food Res. Int. 62 (2014) 424-431.

[31] F. Kayaci, T. Uyar, Encapsulation of vanillin/cyclodextrin inclusion complex in electrospun polyvinyl alcohol (PVA) nanowebs: prolonged shelf-life and high temperature stability of vanillin, Food Chem. 133 (2012) 641-649.

[32] J. Panichpakdee, P. Pavasan, P. Supaphol, Electrospinning of asiaticoside/2-hydroxypropyl- $\beta$-cyclodextrin inclusion complex-loaded cellulose acetate fiber mats: release characteristics and potential for use as wound dressing, Polymer (Korea) 38 (3) (2014) 338-350.

[33] X.Z. Sun, G.R. Williams, X.X. Hou, L.M. Zhu, Electrospun curcumin-loaded fibers with potential biomedical applications, Carbohydr. Polym. 94 (2013) $147-153$.

[34] P. Tonglairoum, T. Chuchote, T. Ngawhirunpat, T. Rojanarata, P. Opanasopit, Encapsulation of plai oil/2-hydroxypropyl- $\beta$-cyclodextrin inclusion complexes in polyvinylpyrrolidone (PVP) electrospun nanofibers for topical application, Pharm. Dev. Technol. 19 (2014) $430-437$.

[35] T. Uyar, Y. Nur, J. Hacaloglu, F. Besenbacher, Electrospinning of functional poly(methyl methacrylate) nanofibers containing cyclodextrin-menthol inclusion complexes, Nanotechnology 20 (2009) 125703.

[36] T. Uyar, J. Hacaloglu, F. Besenbacher, Electrospun polystyrene fibers containing high temperature stable volatile fragrance/flavor facilitated by cyclodextrin inclusion complexes, React. Funct. Polym. 69 (2009) 145-150.

[37] T. Uyar, Y. Nur, J. Hacaloglu, F. Besenbacher, Electrospun polyethylene oxide (PEO) nanofibers containing cyclodextrin inclusion complex, J. Nanosci. Nanotechnol. 11 (2011) 3949-3958.

[38] Z. Aytac, H.S. Sen, E. Durgun, T. Uyar, Sulfisoxazole/cyclodextrin inclusion complex incorporated in electrospun hydroxypropyl cellulose nanofibers as drug delivery system, Colloids Surf. B: Biointerfaces 128 (2015) 331-338.

[39] M.F. Canbolat, A. Celebioglu, T. Uyar, Drug delivery system based on cyclodextrin-naproxen inclusion complex incorporated in electrospun polycaprolactone nanofibers, Colloids Surf. B: Biointerfaces 115 (2014) 15-21.

[40] Z. Aytac, S.I. Kusku, E. Durgun, T. Uyar, Quercetin/ $\beta$-cyclodextrin inclusion complex embedded nanofibers: slow release and high solubility, Food Chem. 197 (2016) 864-871.

[41] Z. Aytac http://www.thesis.bilkent.edu.tr/0006365.pdf.

[42] E.J. Chong, T.T. Phan, I.J. Lim, Y.Z. Zhang, B.H. Bay, S. Ramakrishna, C.T. Lim, Evaluation of electrospun PCL/gelatin nanofibrous scaffold for wound healing and layered dermal reconstitution, Acta Biomater. 3 (2007) 321-330.

[43] G.M. Kim, K.H.T. Le, S.M. Giannitelli, Y.J. Lee, A. Rainer, M. Trombetta, Electrospinning of PCL/PVP blends for tissue engineering scaffolds, J. Mater. Sci.: Mater. Med. 24 (2013) 1425-1442.

[44] E. Luong-Van, L. Grøndahl, K.N. Chua, K.W. Leong, V. Nurcombe, S.M. Cool, Controlled release of heparin from poly ( $\varepsilon$-caprolactone) electrospun fibers, Biomaterials 27 (2006) 2042-2050.

[45] R. Srikar, A.L. Yarin, C.M. Megaridis, A.V. Bazilevsky, E. Kelley, Desorption-limited mechanism of release from polymer nanofibers, Langmuir 24 (2008) 965-974.

[46] T. Higuchi, K.A. Connors, Phase-solubility techniques, Adv. Anal. Chem. Instrum. 4 (1965) 117-212.

[47] A.I. Takahashi, F.J.B. Veiga, H.G. Ferraz, A literature review of cyclodextrins inclusion complexes characterization - Part I: Phase solubility diagram, dissolution and scanning electron microscopy, Int. J. Pharm. Sci. Rev. Res. 12 (2012) 1-6.

[48] K. Harata, Structural aspects of stereodifferentiation in the solid state, Chem. Rev. 98 (1998) 1803-1828.

[49] K. Mishra, H. Ojha, N.K. Chaudhury, Estimation of antiradical properties of antioxidants using DPPH assay: a critical review and results, Food Chem. 130 (2012) 1036-1043.

[50] K. Pyrzynska, A. Pękal, Application of free radical diphenylpicrylhydrazyl (DPPH) to estimate the antioxidant capacity of food samples, Anal. Methods 5 (2013) 4288-4295.

[51] C. Jullian, M. Alfaro, G. Zapata-Torres, C. Olea-Azar, Inclusion complexes of cyclodextrins with galangin: a thermodynamic and reactivity study, J. Solution Chem. 39 (2010) 1168-1177.

[52] Y.P. Neo, S. Ray, J. Jin, M. Gizdavic-Nikolaidis, M.K. Nieuwoudt, D. Liu, S.Y. Quek, Encapsulation of food grade antioxidant in natural biopolymer by electrospinning technique: a physicochemical study based on zein-gallic acid system, Food Chem. 136 (2) (2013) $1013-1021$.

[53] M.E. Carlotti, S. Sapino, E. Ugazio, G. Caron, On the complexation of quercetin with methyl- $\beta$-cyclodextrin: photostability and antioxidant studies, J. Incl. Phenom. Macrocycl. Chem. 70 (2011) 81-90. 